\title{
RESOURCE TOKENIZATION FOR CROWDFUNDING OF WIRELESS NETWORKS
}

\author{
Dr. Volkan Sevindik \\ Digimetrik LLC., 11654 Plaza America Dr., Virginia, 20194, USA \\ NOTE: Corresponding author: vsevindik@gmail.com
}

\begin{abstract}
This paper presents a novel blockchain-based spectrum tokenization method used to crowdsource wireless network deployment projects. Crowdsourcing is a method of financing certain projects and ideas through the funds collected by individuals or businesses in an open marketplace. The method presented in this paper finances the wireless network deployment projects belonging to service providers or governments. The method tokenizes proposed novel wireless resource units, and sells these units to investors. A new Value Unit Per User (VUPU) resource unit is introduced with a new pricing scheme depending on a load of a base station. A novel Proof of Data Load (PoDLO) consensus algorithm is proposed which is used to verify data and traffic load of a base station. Device Diversity Factor (DDF) and Subscriber Unique Permanent Identifier (SUPI) Factor (SUF) are proposed new ways to determine the value of a base station and a network cluster.
\end{abstract}

Keywords - Blockchain, crowdfunding, resource tokenization

\section{INTRODUCTION}

As a telecommunication industry, we are at the verge of deploying $5 \mathrm{G}$ wireless networks. With increased Spectral Efficiency (SE) gains promised by new wireless standards, 5G/NR standard also promises delivering higher SE compared to 4G/LTE. Higher SE enables the transmission of more data to subscribers, increasing revenue for service providers. During network design, service providers calculate how much capacity is needed at certain markets, and coverging the whole market with superior data speeds is the key to success. With mmWave spectrum and with above $6 \mathrm{GHz}$ spectrum bands, reaching to far distances is very challenging, if not impossible. Therefore, wireless operators started to question the investment in 5G technology. Unlimited data plans, decreasing outdoor data usage, and increased competition will force wireless service providers to postpone their infrastructure investments for future wireless network technologies. In the near future, we will see a plateau on wireless data speeds, and innovation because of decreasing network investment.

The other side of the coin is that retail investors or individuals look for more tangible assets to invest. Therefore, a method of investing wireless communication systems' infrastructure can be appealing to the general public. However, wireless network resources should be defined and divided into investable pieces. Tokenization is an important first step of converting a wireless network infrastructure into investable pieces. The open research question is how to tokenize wireless resources so that it provides capital for network investment, and it also provides income to its investors with a minimum risk.

The main purpose of this paper is to share research findings on blockchain-based crowdsourcing platforms. For that purpose, an automated network tokenization method with blockchain is proposed. Section 2 defines novel wireless network resources and a tokenization method, Section 3 defines blockchain and smart contracts used in small-cell blockchain, Section 4 presents newly introduced the Proof of Data Load (PoDLO) consensus algorithm and defines a block creation rate formula, and Section 5 concludes the paper with important next steps.

\section{WIRELESS NETWORK RESOURCES}

\subsection{Network architecture}

Fig. 1 shows 5G network architecture and network interfaces [1]. A base station is connected to a $5 \mathrm{G}$ core network via an N2 interface, and this interface carries all control information from the base station to core network. N3 and N6 interfaces are used to carry user information. When a base station is deployed at a particular location, a backhaul connection is needed to connect to the core network [1]. 


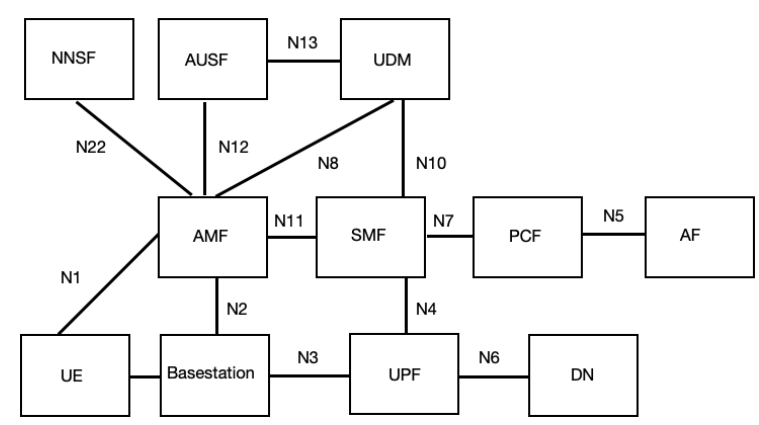

Fig. 1 - 5G network architecture and network interfaces

A backhaul connection can be any type of wireline or wireless connection.

\subsection{Wireless network resource utilization}

\subsubsection{Data tonnage resources}

Spectrum is the most important resource in a wireless network. In this paper, spectrum refers to licensed spectrum. And licensed spectrum is more valuable than unlicensed spectrum. Spectrum is divided into a number of physical resource blocks in 4G/LTE and 5N/NR standards [1],[3-6]. However, depending on the Modulation and Coding Scheme (MCS) selected, the amount of bits that can be carried by one Physical Resource Block (PRB) can change significantly. A resource block is defined as 12 consecutive OFDM symbols in frequency in both $4 \mathrm{G}$ and $5 \mathrm{G}$ standards. One OFDM symbol occupies $15 \mathrm{kHz}$ in frequency in 4G/LTE and this is the same for $5 \mathrm{G} / \mathrm{NR}$ with $15 \mathrm{kHz}$ subcarrier spacing. 5G/NR supports multiple OFDM subcarrier spacings. In 5G/NR, 15, 30, 60, $120 \mathrm{kHz}$ Subcarrier Spacings (SCS) are supported. Thus, one resource block occupies $12 \times 15 \mathrm{kHz}=180 \mathrm{kHz}$ of spectrum, and 10 resource block occupies $1.8 \mathrm{MHz}$ of spectrum in a frequency domain.

$$
N_{P R B, U}=N_{P R B, U}^{1} \cdot \frac{(S A \cdot S S O)}{(12.15) \cdot S C_{F} \cdot\left(N_{S U}\right)}
$$

where $N_{P R B, U}$ represents number of PRBs per subscriber, $N_{P R B, U}^{1}$ is the number of PRBs of subscriber $U$ in 1 msec., SA means total spectrum amount, SSO stands for symbol spacing overhead, $S C_{F}$ is the subcarrier factor for $5 \mathrm{G}$ and $N_{S U}$ is the number of simultaneous users. $S C_{F}$ takes values of 1 , 2,4 , or 8 for $15,30,60,120 \mathrm{kHz}$ SCSs respectively.

$$
T_{U}=N_{P R B, U} \cdot N_{O F D M} \cdot N_{\text {bits }} \cdot M_{U}
$$

where $T_{U}$ means tonnage per subscriber in megabytes (MBs), $N_{P R B, U}$ is the number of PRBs/ subscriber, $N_{O F D M}$ is the number of OFDM symbols per PRB, $N_{\text {bits }}$ is the number of bits per ODFM symbol, $M_{U}$ is the number of connected minutes per user. $\mathrm{SSO}=0.9$, Number of RBs $/ 1 \mathrm{msec} .=2$, Number of Simultaneous Users $=4$, Number of Connected Minutes/User in an hour, which is assumed to be 3 minutes/hour, or 180 seconds/hr. In commercial wireless networks, the simultaneous number of subscriber per Transmission Time Interval (TTI) changes between 2 and 4 for $4 \mathrm{G}$ and between 4 and 8 for 5G. In this article, we assume there are 4 simultaneous subscribers per TTI. And the number of available PRBs is divided by 4 at each TTI.

189 MBs is the unit resource of data tonnage in a wireless network, which is called Value Unit Per User (VUPU). The number of VUPUs will increase when a wireless service provider deploys a network with high-capacity base stations. A wireless provider owning $40 \mathrm{MHz}$ of spectrum, and hundreds of base stations at a particular region will afford more VUPUs than a wireless provider owning $20 \mathrm{MHz}$ of spectrum and a smaller number of base stations. VUPUs will be sold to investors, and this will be the first tokenized unit. The concept of mining using base stations is described in [13]. A base station keeps the record of successfully created/delivered VUPU to each user terminal. And this information is recorded as part of a block in a blockchain held by the base station. Each user is assumed to consume 150 units of VUPU, and these units are tradable. The higher the number of subscribers, the more VUPUs, and the more investment that wireless service provider will have. This will leave more capital for investment, and will motivate service providers to deploy more base stations. Current unlimited data plans cost, on average, $80 \$$ per $20 \mathrm{GBs}$ of data delivered to a subscriber. This equals $80 \$ / 20 \mathrm{~GB}=4 \$ / 1 \mathrm{~GB}=$ $0.4 \$ / 100 \mathrm{MB}$, and this is called Revenue per User (RPU).

$$
\begin{aligned}
& B E T=\max \left\{\left(\sum_{i=180 k}^{T=180(k+1)}\left(R P U_{i} . N\right)\right)-\right. \\
& C o D-C o P, 0\}
\end{aligned}
$$

where BET is the Break Even Time of a base station from a financial perspective, $\mathrm{T}$ is the time that base station is live and in an operational state, $k=0,1,2$, $\mathrm{M} . \mathrm{N}$ is the average number of connected users per base station during time duration of $\{180 \mathrm{k}, 180(\mathrm{k}+1)\}, C o D$ refers to Cost of Base Station Deployment, CoP refers to Cost of Operation, and 
RPU is revenue per connected user, which is calculated at every 15 minutes of connection time. If we assume that one base station capacity is 600 connected users, then total revenue per base station will be 600 Connected Users x $60 \$$. And this calculation assumes that users will stay connected for 24 hours per day and 30 days per month.

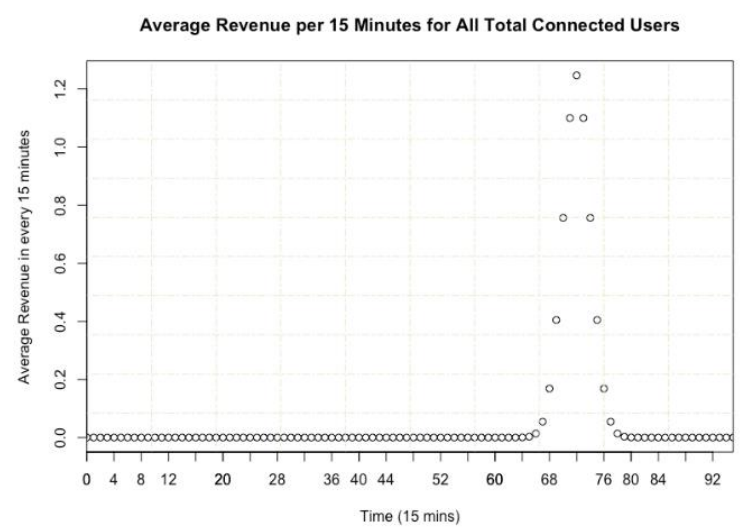

Fig. 2 - Average revenue at each 15 minutes

Fig. 2 shows one sample distribution at busy hours of a day. Fig. 3 shows the revenue of a base station for every 15-minute interval in a day, and one day consists of 9615 -minute intervals. Fig. 5 shows the average revenue per day of a base station.

Fig. 6 shows the average revenue per day for randomly selected three base stations in a network cluster. When a base station is in operational state for a long time, it will be financially faster to breakeven and to reach positive revenue.

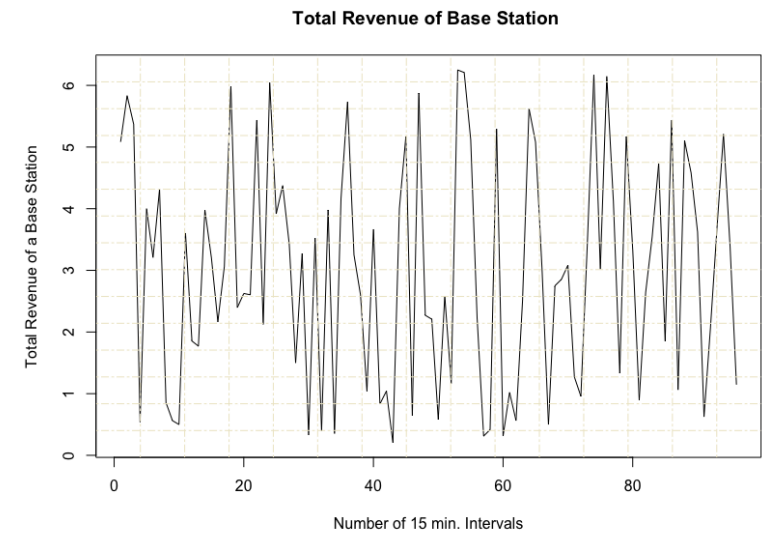

Fig. 3 - Average revenue at each 15 minutes

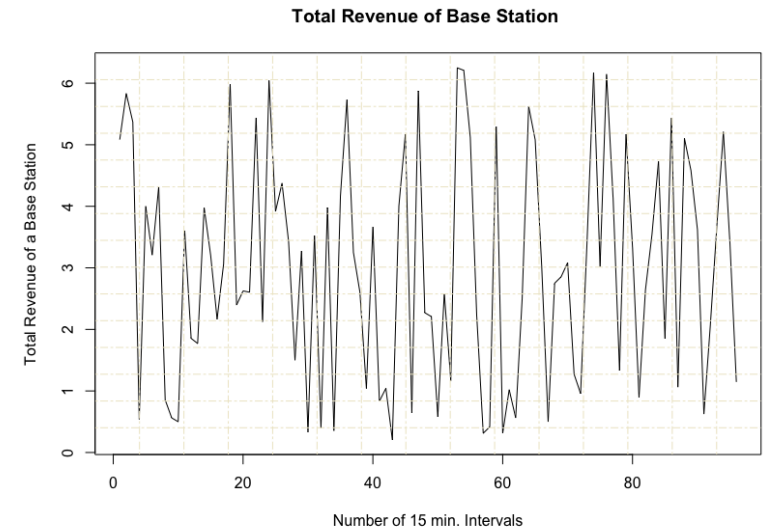

Fig. 4 - Total revenue of base station

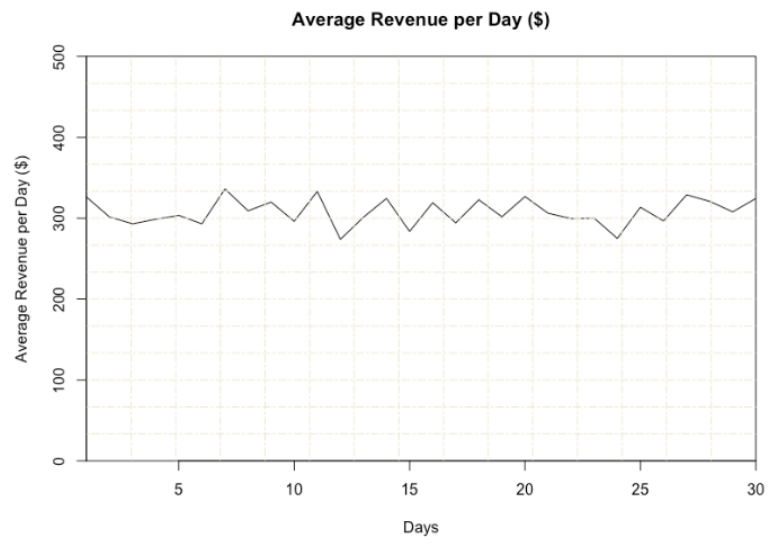

Fig. 5 - Average revenue per day

VUPU investment will start when BET $\neq 0$, and the following formula is used to determine the price of one VUPU:

$$
\begin{aligned}
& P(T)_{\mathrm{VUPU}}= \\
& \frac{\sum_{n=1}^{B}\left(\sum_{i=180 k}^{T=180(k+1)}\left(R P U_{i, n} \cdot N_{n}\right)\right)+\max \{T I, 0\}}{S}
\end{aligned}
$$

where $P(T)_{\text {VUPU }}$ is the price of VUPU at time $T$, and $k$ value ranges from 0 to 959. $S$ is the number of shares issued by the wireless service provider, $B$ is the number of base stations in a certain cluster, or in a certain geographical region.

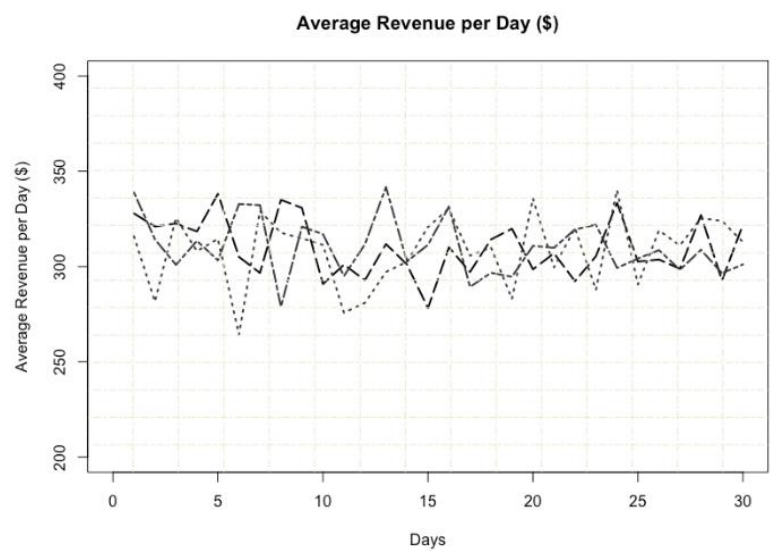

Fig. 6 - Average revenue per day of different base stations 
TI represents the total investment in the same network cluster or in the same geographical region. Since the VUPU price is time dependent, price is updated with the following formula:

$$
\begin{aligned}
P(T)_{\mathrm{VUPU}}= & \left(1-\frac{1}{c}\right) P(T-m)_{\mathrm{VUPU}}+ \\
& \left(\frac{1}{c}\right) P(T)_{\mathrm{VUPU}}
\end{aligned}
$$

where $C$ is the constant used to update the price. With lower $C$ values, a recent calculated price has more impact on the average price, and vice versa. With a newly deployed network cluster, big $C$ values are selected in order to converge to a certain price.

\subsubsection{Mobile device diversity factor}

A base station serves different types of terminals depending on the time and location. A more diverse set of terminals increases the value of the base station, and augments the value of theVUPU. The following formula is used to summarize the impact of terminal diversity:

$$
D D F_{n}=1+\sum_{T T=2}^{Z}\left(\frac{1}{T T}\right)
$$

where $D D F_{n}$ is the device diversity factor of base station $n, T T$ is number of different traffic types served by the base station in a 24-hr window. For example, if the base station serves only one traffic type, $D D F_{n}=1$; and if the base station serves one different traffic type in addition to mobile traffic, then $D D F_{n}=1+0.5=1.5$. Fig. 7 shows DDF values corresponding to different traffic types.

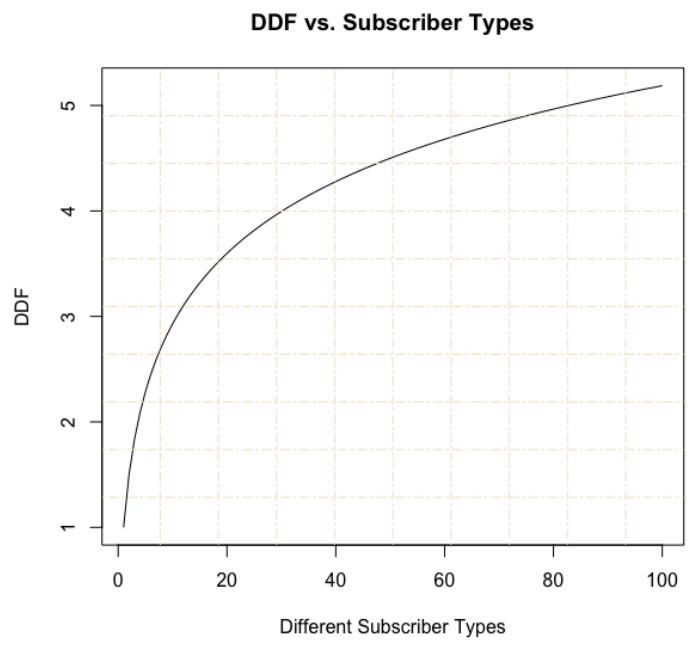

Fig. 7 - Increasing DDF with increading subscriber types
The formula is updated as follows:

$$
\begin{gathered}
P(T)_{\mathrm{VUPU}}= \\
\frac{\sum_{n=1}^{B}\left(D D F_{n} \cdot\left(\sum_{i=180 k}^{T=180(k+1)}\left(R P U_{i, n} \cdot N_{n}\right)\right)\right)+\max \{T I, 0\}}{S} .
\end{gathered}
$$

\subsubsection{Subscriber Unique Permanent Identifier (SUPI) Factor (SUF)}

$S U F_{n}$ means the number of different SUPIs (5G), IMSIs (4G) that are recorded by base station $n$. It is defined as follows:

$$
S U F_{n}=C+\log \left(N_{S U P I}\right) .
$$

If $S U F_{n}=1$, this means the same users connect to the same base station n. For modeling the SUPI factor, any logaritmic function can be used. The reason for using the logarithmic function is that the increasing number of diversity in the network will have lower impact on the value since the base station will be overloaded after some point leading the total service quality degradation for all terminals connected to the base station. In a commercial network, user capacity per base station ranges between 600 and 1200 connected users for $5 \mathrm{G}$ base stations. And, with SUF $=1$, there are 600 different SUPIs recorded by the base station. If SUF $=2$, this means there are 1200 unique SUPIs recorded by the base station. With SUF, the new formula for the price of VUPU will be

$$
\begin{aligned}
& P(T)_{\mathrm{VUPU}} \\
& \quad \frac{\sum_{n=1}^{B}\left(D D F_{n} \cdot S U F_{n} \cdot\left(\sum_{i=180 k}^{T=180(k+1)}\left(R P U_{i, n} \cdot N_{n}\right)\right)\right)}{S^{\left(\frac{1}{D D F . S U F}\right)}} \\
& \quad+\frac{\max \{T I, 0\}}{S^{\left(\frac{1}{D D F . S U F}\right)}} \cdot
\end{aligned}
$$

The DDF value curve can have any shape depending on the number of hand-offs. Fig. 8 shows the DDF of a base station serving both mobile and nomadic users. Since subscribers/users are mobile, subscribers/users will hand off from one base station to another base station during the day. When users are stationary or nomadic, depending on the location of the user, Wifi might be available. Thus, users will use Wifi connection especially on these occasions. This shows that the real value of a cellular communication system is to provide high data speeds during mobility or in other words, to provide mobile data to mobile users. 


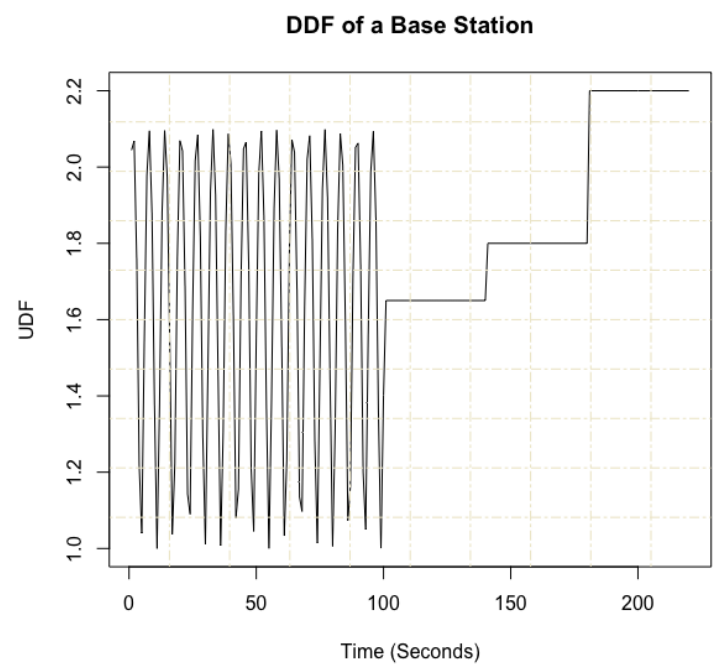

Fig. 8 - DDF of a base station based on mobility

Fig. 9 shows real wireless network deployment. Base station 1 and base station 2 are co-located, and they provide coverage using different operational spectrum. This model is called the 'overlay' coverage method, where one base station uses a lower frequency band to provide greater coverage than a co-located base station using a mid or high frequency band. The higher the mobility in the network, the higher the value that network delivers to its subscribers. Hence, the DDF factor is included in the pricing model since the network will be more valuable if there are a high number of mobile users or highly mobile users in the network.

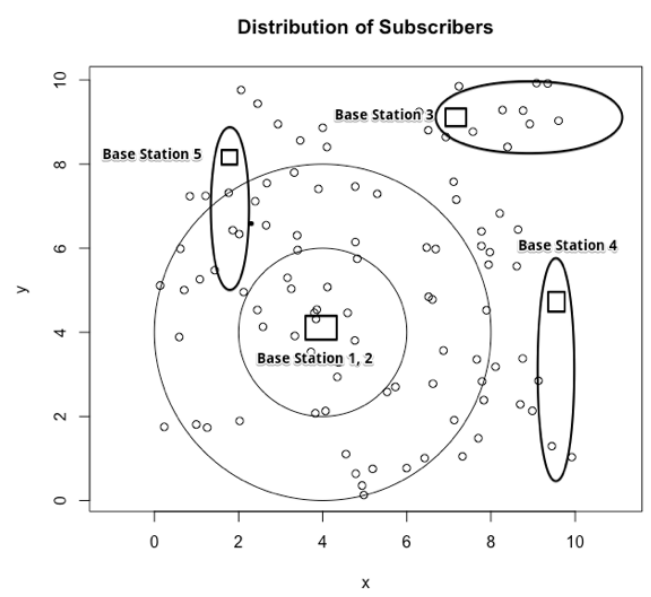

Fig. 9 - Distribution of subscribers In a real network cluster

\section{BLOCKCHAIN}

\subsection{What is blockchain?}

Blockchain is a distributed ledger technology that records transactions securely in an untrusted system [2], [13]. Blockchain records each transaction as a part of an existing block or as a new block. The first block in a blockchain is called the genesis block, and this is where blockchain starts. Each block generates encrypted input to the next block, and this creates extremely secure chains of blocks, where one unauthorized change in a block will make all blocks invalid [2], [12-13].

Each block in a blockchain is generated by a group of computing nodes called miners [2]. Miners collaboratively generate each block by following strict rules of generating a block, and by using strong cryptographic hash algorithms such as SHA-256 [10], [13]. Each VUPU created is recorded in a blockchain and any transaction performed on VUPU is also recorded in a blockchain. A transaction can be a sell transaction, a buy transaction, or an exchange transaction. Since VUPUs are recorded in blockchain, it is impossible to change any transaction details.

\subsection{Small-cell blockchain}

In a small-cell blockchain, each event in the network is recorded as a part of an existing block or part of a new block [2], [12]. An event in the network is defined as any action occurring in the network. For instance, a newly changed small-cell parameter is an event, or the amount of data transmitted to a user in the downlink is also an event. The higher the number of small-cells, the higher the number of events happening in the network; however, block generation will be also faster since a high number of small-cells will mine a block in small-cell blockchain [2].

\section{BASE STATION TOKENS}

The formula for the price of VUPU is

$$
\begin{aligned}
& P(T)_{\text {VUPU }} \\
& =\frac{\sum_{n=1}^{B}\left(D D F_{n} \cdot S U F_{n} \cdot\left(\sum_{i=180 k}^{T=180(k+1)}\left(R P U_{i, n} \cdot N_{n}\right)\right)\right)}{S\left(\frac{1}{D D F \cdot S U F}\right)} \\
& \quad+\frac{\max \{T I, 0\}}{\left.S^{\left(\frac{1}{D D F} \cdot S U F\right.}\right)} .
\end{aligned}
$$


The first set of shares of a cluster will be 1000 shares, and issue time will depend on network performance. Naturally, shares will be issued when they are the most valuable, and also shares will be sold by investors when they are the most valuable. Fig. 10 shows the impact of DDF on the number of distributed shares of a cluster. When DDF increases, the value of the network cluster will increase and the price of the issued share will also increase. If the wireless network serves a diverse set of traffic at the beginning of network operation, then the share price will increase faster and will stabilize later (DDF $=2,3$ ). An additional set of traffic served by the network will also increase the price, however with a decreasing step-size (DDF $=4)$. In this article, the demand of investors on the price of shares is not investigated, and it will be studied in the next article. Each network cluster has a cluster head. When VUPUs are issued for a network cluster, VUPUs are recoded in small-cell blockchain as part of an existing block or as a new block. Blocks are generated by each miner base station in a network cluster, and each network cluster has a different number of miners. A consensus algorithm is used to verify the load of each base station in the network. Proof of work is used in bitcoin network [2], and Proof of stake is used in an Ethereum network [12].

The Proof of Data Load (PoDLO) consensus algorithm is a newly introduced consensus algorithm and is used to verify the load of a base station so that the correct VUPUs are calculated. A cluster head sends load information to each base station in the cluster, and there might be a direct connection between base stations. If there is no direct connection between two base stations, messages are relayed through base station(s) in the middle.

There is a total of 6 messages exchanged between base stations for a network shown in Fig. 11. Fig. 12 shows that there are $2 . \mathrm{N}+\mathrm{N} \cdot(\mathrm{N}-1)=\mathrm{N} .(\mathrm{N}+1)$ messages exchange between nodes, where $\mathrm{N}$ is the total number of base stations except the cluster head. This is a logical network architecture with one cluster head controlling two base stations. Since each VUPU transaction is recorded in a block, the block creation rate is very crucial. And the block is created when the network cluster has received votes from each base station.

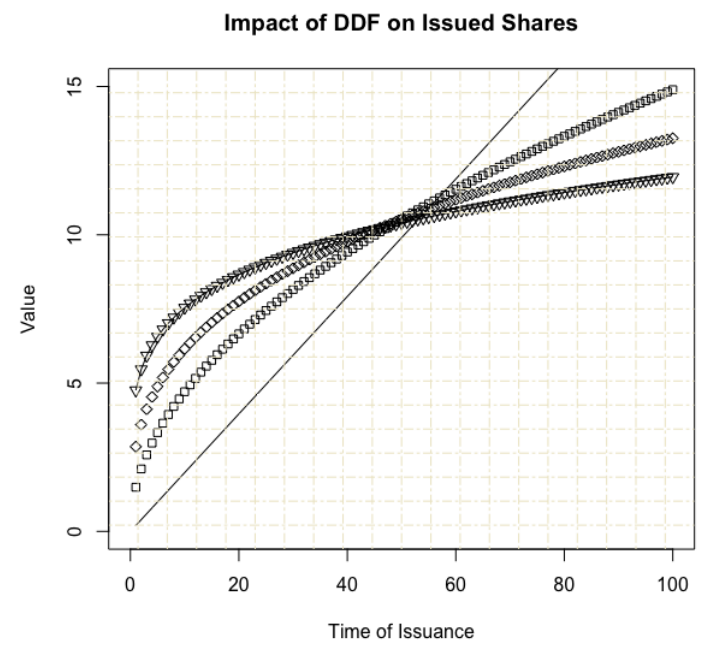

Fig. 10 - Impact of DDF on the issued VUPU shares

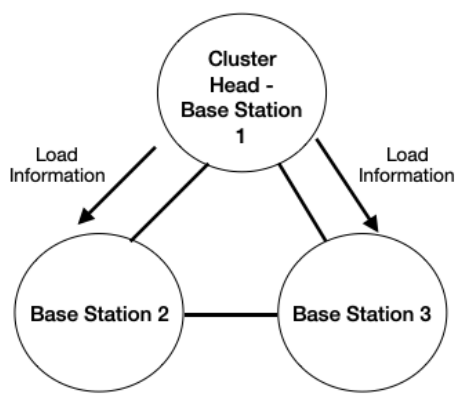

Fig. 11 - Cluster head, base stations, and messaging

Vote means 'load verification' messages that each base station sends to the network cluster. There is one cluster head running the consensus algorithm, and each base station belonging to the cluster sends its load verification votes to the network cluster head. Load verification means the verifying of a load of a base station in the network by another base station so that accurate load information can be collected for better price calculation of network resources. When a base station verifies a load of another base station, the verifier base station sends a 'Yes' vote to the cluster head, and otherwise the verifier base station sends a 'No' vote to the cluster head. If the number of 'Yes' votes is larger than 'No' votes, then a new block is created that is used to record all VUPUs related network transactions.

$$
B C R=(C \cdot M \cdot D T L)+\left(B P S^{\left(\frac{1}{T D F}\right)}\right)
$$

where $\mathrm{BCR}$ is the block creation rate, $\mathrm{C}$ is the constant, $\mathrm{M}$ is the number of nodes except the cluster head, DTL is data transmission latency, and BPS is the base station's processing speed. BPS can be represented with a logarithmic function since processing speed goes down with an increasing 
number of processes. Each base station consists of central processing unit(s), and memory units the power consumption of which increases with the increasing amount of load. Increasing power consumption also increases the heat on the device, which in turn decreases the processing capability of the device.

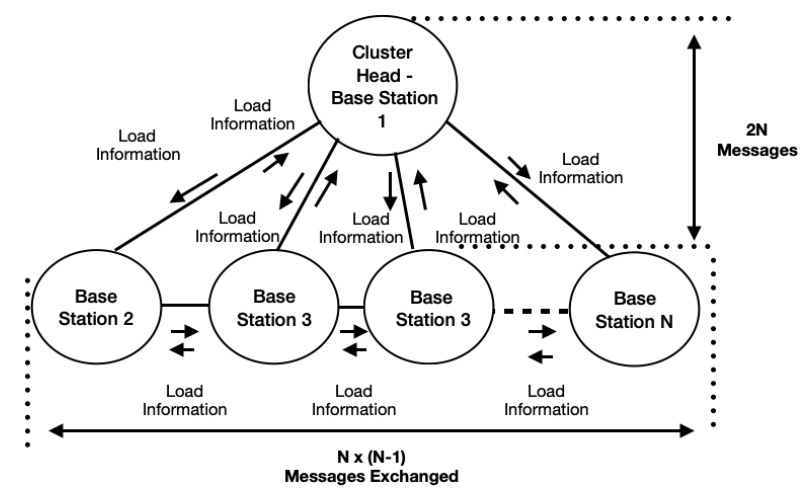

Fig. 12 - Network cluster message exchange model

One proposed model for BPS is

$$
B P S=\left(\frac{1}{a}\right) \cdot\left(V^{\left(\log _{10}\left(\frac{V}{M}\right)\right)}\right)+C
$$

where ' $a$ ' is the number of processors of base station hardware, or number of cores in a single processor. ' $\mathrm{V}$ ' is the number of processes, threads processed by hardware of the base station and ' $C$ ' is the number of processors or processor cores assigned to the mining process.

Fig. 13 shows data processing latency, that is (1-DPS), of a base station with one processor $(\mathrm{a}=1)$, two processors $(\mathrm{a}=2)$, and four processors $(\mathrm{a}=4)$. $\mathrm{M}$ is the constant related to the CPU model, speed, and impact of heat on CPU performance. $\mathrm{M}$ is a direct function of CPU processing power/speed, and the load on a CPU. With increasing CPU load and the data processing speed, $\mathrm{M}$ will also increase.

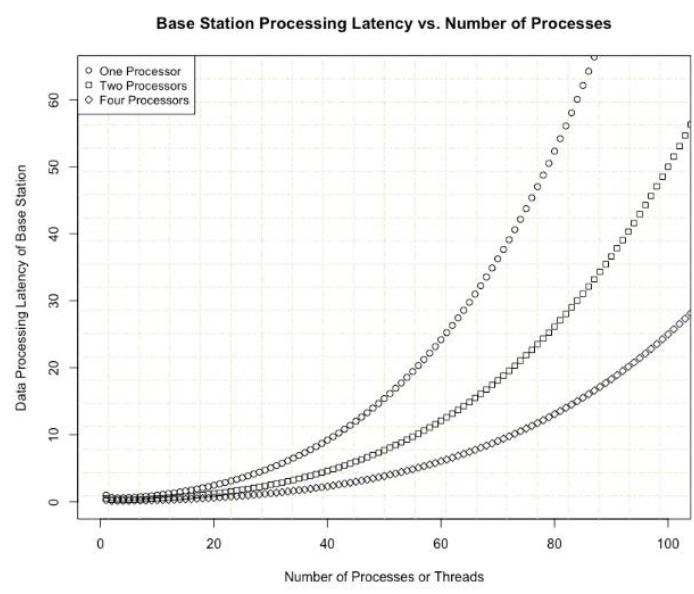

Fig. 13 - Base station processing latency
$M$ value is usually given by the manufacturer of the CPU and it is unique to CPU model, type, and family. When a certain percentage of a base station's resources is assigned to the mining process, BCR will increase since miner base stations will only use these resources to create a block in small-cell blockchain. LTDS depends on the type of the link changing between (1msec., 10 msec.). With an increasing number of nodes, BCR also increases. The VUPU transaction rate will decrease which is not acceptable since a fast VUPU transaction record rate should be very fast so that buy and sell orders can be executed in almost real time. On the contrary, bigger $\mathrm{N}$ values will lead to a higher VUPU value. With one cluster head in a network cluster with 100 base stations each with a single processor, with $\mathrm{TDF}=2.5$, the $\mathrm{BCR}$ is calculated as follows:

$$
\begin{gathered}
B C R=(2 \times 100 \times 10 \mathrm{msec})+\left(\text { DPS }\left(\frac{1}{2.5}\right)\right)= \\
2 \mathrm{sec}+40 \mathrm{msec}=2.04 \mathrm{sec} .
\end{gathered}
$$

By changing the cluster size, base station type, transmission link type between base stations, BCR can be changed, adjusted and be made location and time dependent.

\section{CONCLUSION}

This paper presented a novel blockchain-based tokenization method for wireless network resources. A tokenization method tokenizes network reources, and determines the price of each unit of these resources. Tokenized network resources can be transacted by network investors, and collected funds can be used by service providers to finance new $5 \mathrm{G}$ and $6 \mathrm{G}$ network deployment.

This crowdfunding method enables network investment by individuals and companies. VUPU, DDF and SUF terms are newly introduced in this paper. The price of VUPUs can be calculated differently depending on how fast the investment fund should be sourced. A new formula to calculate the price of VUPU is disclosed. With increasing DDF and SUF values, the VUPU price also increases. VUPUs can be bought and sold at any time, and each VUPU transaction is recorded in a blockchain. 


\section{REFERENCES}

[1] 3GPP Technical Standard Release 16. Available:https://www.3gpp.org/release-16

[2] Bitcoin: A Peer-to-Peer Electronic Cash System. Available: https://bitcoin.org/bitcoin.pdf

[3] J. Wang, J. Weitzen, V. Sevindik, O. Bayat, M. Li, "Dynamic Centralized Interference Coordination in Femto Cell Network with QoS Provision", Proceedings of the 18th International Conference on Communications (CSCC '14), pp. 81-86, July 2014

[4] V. Sevindik, Wireless Spectrum Trading: Techniques and Key Performance Indicators, Amazon Digital Services LLC, 2016

[5] V. Sevindik, Systems and methods for configuring a scheduler at an access node, US Patent No: 9894677

[6] V. Sevindik, Systems and methods for scheduling transmissions from an access node, US Patent No: 9525535

[7] V. Sevindik, J. Wang, O. Bayat, J. Weitzen, "Performance Evaluation of a Real Long Term Evolution (LTE) Network", 37th Annual IEEE Conference on Local Computer Networks", Fenruary 2013.

[8] V. Sevindik, O. Bayat, J. Weitzen, "Radio Resource Management and packet scheduling in femtocell networks", International Symposium of Modeling and Optimization of Mobile, Ad Hoc and Wireless Networks, May 2011.

[9] 3GPP Specification \#36.423. Available: https://portal.3gpp.org/desktopmodules/Sp ecifications/SpecificationDetails.aspx?specifi cationId $=2452$
[10] Cryptographic Hash Function. Available: https://en.wikipedia.org/wiki/Cryptographi c hash function

[11] 3GPP Specification \#36.410. Available: https://portal.3gpp.org/desktopmodules/Sp ecifications/SpecificationDetails.aspx?specifi cationId $=2443$

[12] Ethereum Smart Contract. Available: https://www.ethereum.org/

[13] Smallcellcoin. Available: https://www.smallcellcoin.com

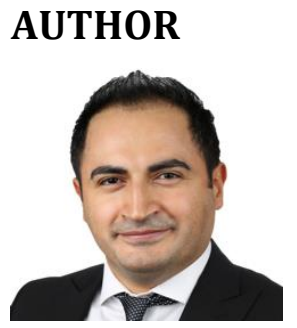

Dr. Volkan Sevindik received a B.S. degree in electronics and telecommunication engineering with distinction (Summa Cum Laude) from Istanbul Technical University, Istanbul, Turkey, and an M.S. degree in electronics and electrical engineering from Bogazici University, Istanbul, Turkey, and a Ph.D. degree in electrical and computer engineering from University of Massachusetts, Lowell, MA, USA. Dr. Sevindik has held numerous managerial, and technical consultant roles in tier-1 wireless service operators, and well known consulting companies all over the world. He is the founder of Smallcellcoin Inc., where he has been conducting research on blockchainbased automated wireless network deployment, and management techniques since 2016. Dr. Sevindik holds 35 granted US patents with a total of 62 US patent applications. He has published 23 conference and journal papers, 1 book, 2 book chapters. He is an active member of IEEE, and he is actively providing technical consulting services on wireless network architecture, network financial planning, spectrum acquisition, and pricing. 